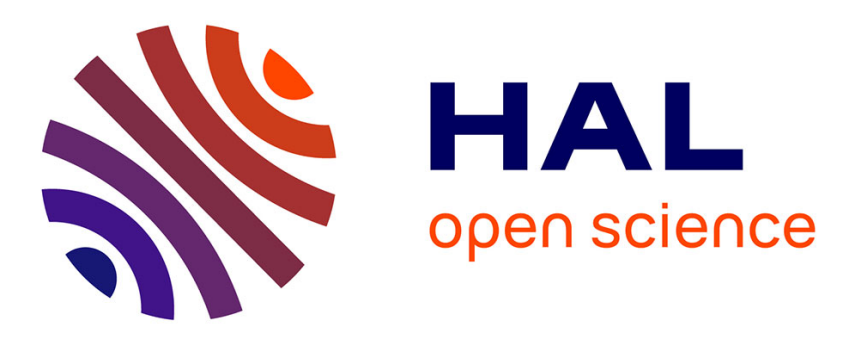

\title{
Application of Spectral Statistics to Spectral Texture Discrimination
}

\author{
Hilda Deborah, Noël Richard, Jon Yngve Hardeberg
}

\section{To cite this version:}

Hilda Deborah, Noël Richard, Jon Yngve Hardeberg. Application of Spectral Statistics to Spectral Texture Discrimination. 2018 Colour and Visual Computing Symposium (CVCS), Sep 2018, Gjøvik, Norway. pp.1-6, 10.1109/CVCS.2018.8496628 . hal-02407264

\section{HAL Id: hal-02407264 \\ https://hal.science/hal-02407264}

Submitted on 12 Dec 2019

HAL is a multi-disciplinary open access archive for the deposit and dissemination of scientific research documents, whether they are published or not. The documents may come from teaching and research institutions in France or abroad, or from public or private research centers.
L'archive ouverte pluridisciplinaire HAL, est destinée au dépôt et à la diffusion de documents scientifiques de niveau recherche, publiés ou non, émanant des établissements d'enseignement et de recherche français ou étrangers, des laboratoires publics ou privés. 


\title{
Application of spectral statistics to spectral texture discrimination
}

\author{
Hilda Deborah*, Noël Richard ${ }^{\dagger}$, and Jon Yngve Hardeberg ${ }^{\ddagger}$ \\ *, ${ }_{\text {Department }}$ of Computer Science, Norwegian University of Science and Technology \\ Teknologiveien 22, Gjøvik 2815, Norway \\ ${ }^{\dagger}$ Laboratory XLIM, JRU CNRS 7252, University of Poitiers \\ 11 Bd Marie et Pierre Curie, Futuroscope Chasseneuil Cedex 86962, France \\ *hilda.deborah@ntnu.no, †noel.richard@univ-poitiers.fr, $\ddagger_{j}$ jon.hardeberg@ ntnu.no
}

\begin{abstract}
Hyperspectral imaging has continued to be exploited in various fields for its offer of gain in accuracy, despite the cost and complexity of its acquisition. However, accuracy must be maintained in all processing chain for the potential to be optimally exploited. A spectrum is a continuous function over the wavelengths and it must be processed as such. Taking that into consideration, in this article, a statistical processing of hyperspectral images is proposed. The statistics is based on the Kullback-Leibler pseudo-divergence measure, which incorporates the mathematical definition of a spectrum as a continuous function. The interest of these statistics is then demonstrated through a task of spectral texture discrimination.
\end{abstract}

Index Terms-Hyperspectral, texture, Mahalanobis distance, Kullback-Leibler pseudo-divergence

\section{INTRODUCTION}

Hyperspectral imaging (HSI) was originally developed for the earth observation and remote sensing fields [1]. However, nowadays, it has garnered attentions from a wide variety of disciplines and applications. HSI has been employed to assess the quality and safety of food products due to its offer of speed, accuracy, and reliability [2], [3]. By coupling a hyperspectral imager and a microscope, a multiscale method was proposed for noninvasive detection of cervical cancer. Its results were consistent with those of hystopathological analysis, demonstrating accuracy and efficacy [4]. In wood product manufacturing, where logs need to be sorted according their moisture content (MC), HSI has been used to generate MC model with reasonable accuracy [5]. It has also been largely exploited in precision agriculture due to its spectral range and precision to profile materials and organisms [6].

The common reason found in the aforementioned uses of HSI is its accuracy, precision, and reliability. In other words, it has been increasingly exploited due to its potential and capability in adhering to metrological constraints. And this is despite its costly and complex acquisition. However, an accurate measurement of scene or surface does not automatically entail an accurate and relevant final processing result. The metrological aspects, e.g., bias and uncertainty [7],

This work has been partly supported by French national projects ANR DigiPi (driven by XLIM and CRC), the European Union, and the region of Nouvelle Aquitaine (ERDF NUMERIC program, action e-Patrimoine). of hyperspectral data have to be maintained throughout the subsequent processing steps.

With the end goal of developing a full metrological framework of hyperspectral image analysis and processing, we have carried out extensive works on more basic levels, i.e., spectral difference functions [8], [9], spectral ordering relations [10], and elementary operations for spectral mathematical morphology [11]. In this study, we are introducing our next endeavor on the framework development, specifically on statistics/ moments for hyperspectral data. The proposed spectral statistics are built based on Kullback-Leibler pseudo-divergence (KLPD) [9] and their potential will be demonstrated in a preliminary study of spectral texture assessment.

The rest of the article is organized as follows. Section II provides a recall to the definition of a spectrum being used in our study. As a note, the content of this section has first appeared in [12]. The proposed spectral statistics are provided in Section III, along with brief explanations of its building blocks. Applications of the proposed statistics are provided in two parts. Section IV provides a demonstration of how the statistics can be used to model the spectral content of texture images. Then, in Section V, average spectral Mahalanobis distance is employed to determine the similarity between pairs of texture images. This texture discrimination application exploits the knowledge obtained through the modeling of spectral content provided in Section IV. Finally, Section VI provides the concluding remarks for this article.

\section{Spectral Measure, Spectral Function, And SPECTRAL SignAL}

Under the point of view of signal and image processing, a spectrum is a signal that is associated to a mathematical model of a continuous function over the wavelength $\lambda$ [9], [11]. It expresses the acquired energy coming from a surface, scene, or light source. This makes a spectrum directly related to the physical and optical properties of the objects. Furthermore, under this physical point of view, a spectrum is positive since it is related to energy (radiance, in $W^{-2} s r^{-1}$ ) or its ratio (reflectance, in percentage).

In remote sensing applications, we can find discontinuities in several wavelengths due to atmospheric corrections, e.g., 
water absorption around 1200 and $1450 \mathrm{~nm}$. Thus, a spectrum $S$ can be more specifically defined as a continuous function over the wavelengths $f(\lambda)$, that is of $C^{0}$ class function to say that $S$ is not differentiable everywhere. Finally, a hyperspectral acquisition of a continuous spectrum can be regarded as a spectral sampling of the continuous physical spectrum $S(\lambda)$, generating a sequence of measurements $s\left(k \cdot \delta_{\lambda}\right)$ (Eq. 1).

$$
s\left(k \cdot \delta_{\lambda}\right)=\left\langle f(\lambda), \zeta\left(\lambda-k \cdot \delta_{\lambda}\right)\right\rangle
$$

The spectral sampling is performed using optical filters $\zeta(\lambda)$ upstream the photon detector, justifying the use of scalar product. $\zeta(\lambda)$ is generally approximated by a Gaussian or trapezoidal functions and is characterized by the Full Width Half Max (FWHM).

Due to the sequence of measures $s\left(k \cdot \delta_{\lambda}\right)$, a spectrum has often been implicitly considered as a set of independent measures. It is then being used as a vector, probability density function, or sequence [8]. Consequently, L2 norm and its derived forms and also divergence functions are often used to assess differences or similarities between spectra. Such similarity constructions are at the core of strategies commonly used for hyperspectral data, e.g., dimensionality reduction.

In the rest of this article, a spectrum will be considered in its continuous expression. However, readers are asked to keep in mind the spectral sampling $\delta_{\lambda}$ of the digitization, derivation, and other basic digital signal processing understanding.

\section{Spectral Difference-Based Statistics}

In this work, a spectrum $S$ is defined as the realization of a random variable $\mathcal{S}$. The mathematical definition of a spectrum and the choice of distance/ difference measure cannot be separated [8]. Thus, the measure to choose must be one that considers $S$ as a continuous function rather than, e.g., as a vector. Once the choice has been made, more advanced analysis and processing tools will become available, e.g., mathematical morphology, nonlinear filtering, and classification.

\section{A. Kullback-Leibler pseudo-divergence}

Kullback Leibler pseudo-divergence (KLPD) [9] was developed specifically for hyperspectral data, taking into account its continuous expression. Strictly speaking, KLPD is not a distance function since it does not hold triangular inequality criterion. However, it passes the metrological protocol developed in [8]. KLPD $d_{\widetilde{K L}}$ measures the difference between two arbitrary spectra $S_{1}$ and $S_{2}$ through the formula in Eq. 2. As stated, KLPD is composed of two components, i.e., differences in shape $\Delta G$ and intensity or energy level $\Delta W$.

$$
\begin{gathered}
d_{\widetilde{\mathrm{KL}}}\left(S_{1}, S_{2}\right)=\Delta G\left(S_{1}, S_{2}\right)+\Delta W\left(S_{1}, S_{2}\right) \\
\Delta G\left(S_{1}, S_{2}\right)=k_{1} \cdot d_{\mathrm{KL}}\left(\bar{S}_{1}, \bar{S}_{2}\right)+k_{2} \cdot d_{\mathrm{KL}}\left(\bar{S}_{2}, \bar{S}_{1}\right) \\
\Delta W\left(S_{1}, S_{2}\right)=\left(k_{1}-k_{2}\right) \log \left(\frac{k_{1}}{k_{2}}\right)
\end{gathered}
$$

The construction of KLPD is based on information divergence using Kullback-Leibler (KL) divergence [13]. KL divergence $d_{\mathrm{KL}}$ is, however, designed to measure similarity between two probability density functions. And to use it for

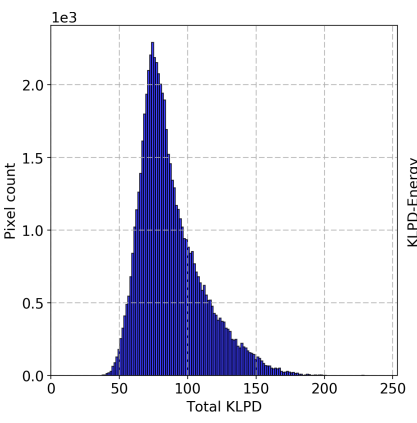

(a) HSD-1D histogram

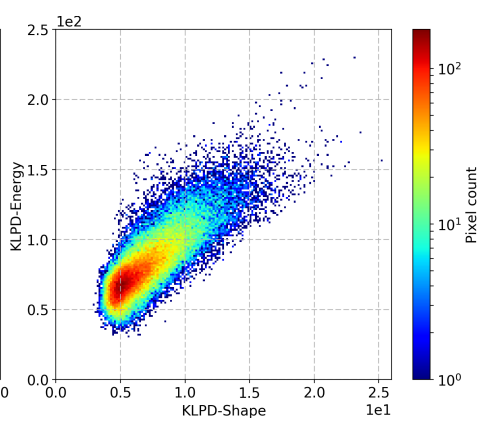

(b) BHSD-2D histogram
Fig. 1. Histogram of spectral differences (HSD) computed using KLPD difference function and bidimensional histogram of spectral differences (BHSD) obtained by separating the shape and energy components of KLPD.

spectra, the spectra must satisfy the constraint of probability density functions, i.e., their integrals must be equal to 1 . Thus, the input to $d_{\mathrm{KL}}$ are normalized spectra $\bar{S}_{1}$ and $\bar{S}_{2}$, see Eq. 3 .

$$
\begin{gathered}
d_{\mathrm{KL}}\left(\bar{S}_{1}, \bar{S}_{2}\right)=\int_{\lambda_{\min }}^{\lambda_{\max }} \bar{S}_{1}(\lambda) \cdot \log \frac{\bar{S}_{1}(\lambda)}{\bar{S}_{2}(\lambda)} d \lambda \\
\bar{S}=\left\{\bar{s}(\lambda)=\frac{s(\lambda)}{k}, \forall \lambda \in\left[\lambda_{\min }, \lambda_{\max }\right]\right\} \\
k=\int_{\lambda_{\min }}^{\lambda_{\max }} s(\lambda) d \lambda
\end{gathered}
$$

\section{B. Bidimensional histogram of spectral differences}

The distribution of a spectral set $\mathcal{S}$ can be observed through the histogram of differences $\left\{d_{\widetilde{\mathrm{KL}}}\left(S_{i}, S_{\mathrm{ref}}\right), \forall S_{i} \in \mathcal{S}\right\}$, where $S_{\text {ref }}$ is any arbitrary spectral reference. As an illustration, spectral differences of image parchment-P1 (Fig. 3e) are obtained using KLPD function and a theoretical equi-energetic white spectrum as reference. The histogram of spectral differences (HSD) is as shown in Fig. 1a.

The fact that KLPD is composed of two independent shape and energy components allows expanding the HSD into a two-dimensional one. The bidimensional histogram of spectral differences (BHSD) of the same image using the same spectral reference is plotted in Fig. 1b. In this representation, the $\mathrm{x}$ and y-axes display spectral variations in shape and intensity, respectively. The origin of BHSD is always of the chosen spectral reference $S_{\text {ref }}$, which partially dictates the distribution of spectral differences of any given image. However, the discussion of spectral reference selection is out of the scope of this article and, instead, can be read in Ref. [11].

\section{Spectral variance-covariance matrix}

By defining a spectrum $S$ as the realization of random variable $\mathcal{S}, d_{\widetilde{\mathrm{KL}}}\left(S, S_{\text {ref }}\right)$ can therefore be considered as a random process (or a multivariate one) with two components $\Delta G$ and $\Delta W$. This further allows analyzing the diversity of $\mathcal{S}$ relative to a spectral reference $S_{\text {ref }}$, through variancecovariance matrix $\widetilde{\Gamma}_{\mathcal{S}, S_{\text {ref }}}$, see Eq. 4 and 5 .

$$
\widetilde{\Gamma}_{\mathcal{S}, S_{\text {ref }}}=\left(\begin{array}{ll}
\alpha_{G G, S_{\text {ref }}} & \alpha_{G W, S_{\text {ref }}} \\
\alpha_{G W, S_{\text {ref }}} & \alpha_{W W, S_{\text {ref }}}
\end{array}\right)
$$




$$
\begin{aligned}
\alpha_{G G, S_{\mathrm{ref}}} & =\sum_{S i}\left(\Delta G\left(S_{i}, S_{\mathrm{ref}}\right)\right)^{2} f\left(S_{i}\right) \\
\alpha_{W W, S_{\text {ref }}} & =\sum_{S i}\left(\Delta W\left(S_{i}, S_{\mathrm{ref}}\right)\right)^{2} f\left(S_{i}\right) \\
\alpha_{G W, S_{\mathrm{ref}}} & =\sum_{S i} \Delta G\left(S_{i}, S_{\mathrm{ref}}\right) \Delta W\left(S_{i}, S_{\mathrm{ref}}\right) f\left(S_{i}\right)
\end{aligned}
$$

$\widetilde{\Gamma}_{\mathcal{S}, S_{\text {ref }}}$ can be further developed into spectral inner variancecovariance matrix when the median $\widetilde{\mu}_{\mathcal{S}}$ of a spectral set $\mathcal{S}$ is employed as the reference $S_{\text {ref }}$. The variance-covariance matrix

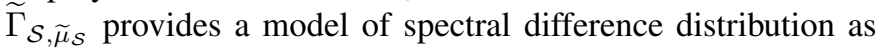
a bivariate normal law.

\section{Spectral Mahalanobis distance}

Spectral variance-covariance matrix introduced in the previous section further allows computing spectral Mahalanobis distance (MD) between a given spectrum $S$ to the distribution of $\mathcal{S}$ in the histogram of spectral differences. The mathematical expression to MD is provided in Eq. 6.

$$
d_{\mathrm{M}}(S, \mathcal{S})=\sqrt{\left(x_{S}-\widetilde{\mu}_{\mathcal{S}}\right)^{T} \widetilde{\Gamma}_{\mathcal{S}, \tilde{\mu}_{\mathcal{S}}}\left(x_{S}-\widetilde{\mu}_{\mathcal{S}}\right)}
$$

This expression provides a natural definition of the distribution of $\mathcal{S}$ through the use of its median $\widetilde{\mu}_{\mathcal{S}}$ and variance-covariance

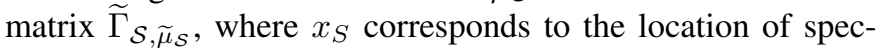
trum $S$ in the BHSD space computed relative to $\widetilde{\mu}_{\mathcal{S}}$.

As a final note, in these spectral statistics, a median spectrum $\tilde{\mu}_{\mathcal{S}}$ instead of an average one $\bar{\mu}_{\mathcal{S}}$ has been used to model the spectral expectation $E[S]$. This is because to define a spectrum of local average $\bar{\mu}_{\mathcal{S}}$, a marginal average of spectral values is required, in which the natural interdependency between spectral channels are lost.

\section{SPeCtral Statistics For Modeling Spectral TeXture}

The inner nature of textured surfaces or objects induces an important variability of spectra within a spectral image. Several approaches are available to measure spectral variability [14]-[16], including statistical models [17], [18]. However, in these statistical approaches, a spectrum is considered as a vector. In the following, we will use the proposed spectral statistics to model spectral variability within a spectral image.

\section{A. Experimental setup and dataset}

Experimental setup for using the proposed spectral statistics to model spectral variability of a set or image can be seen in Fig. 6. Note that the computation of spectral differences is only carried out once. But since KLPD function is composed of two independent components, inner BHSD representation is also obtained in addition to the total KLPD map.

11 texture images of size $250 \times 250$ pixels are used in this study, see Fig. 3. Each of them is of 186 spectral channels, in the range of $405.37-995.83 \mathrm{~nm}$, in $3.26 \mathrm{~nm}$ interval. The dataset consists of man-made texture coming from textile and commercial grade parchment and papyrus, and objects coming from the nature, i.e., leaves, rose petal, moss, and lichen.

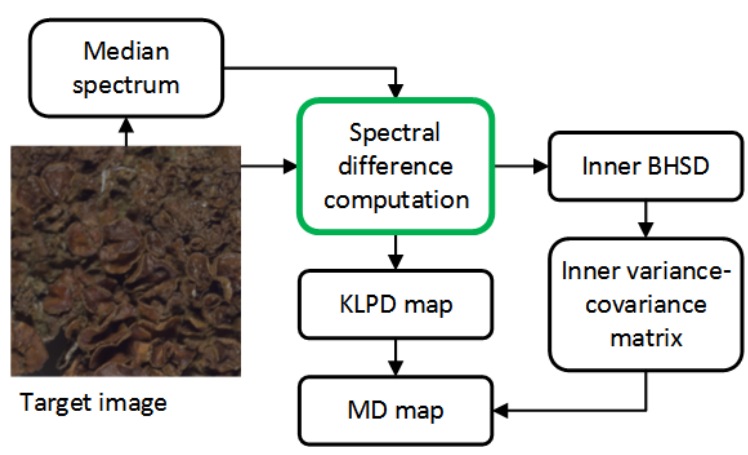

Fig. 2. Experiment workflow of the use of proposed spectral statistics for modeling spectral variability of an image. Spectral difference computation is carried out once, providing both KLPD map and inner BHSD representation.

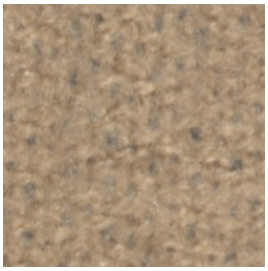

(a) textile-T1

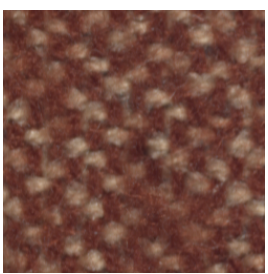

(d) textile-T4

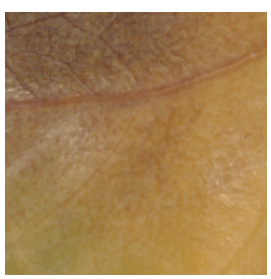

(g) leaf-L1

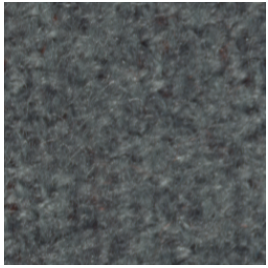

(b) textile-T2

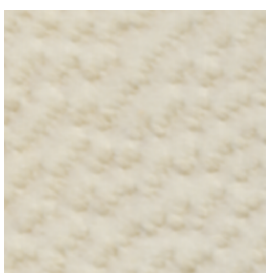

(e) parchment-P1

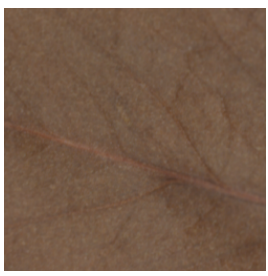

(h) leaf-L2

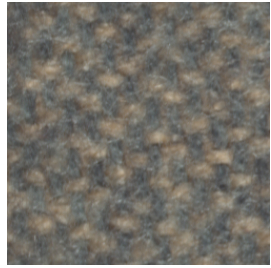

(c) textile-T3

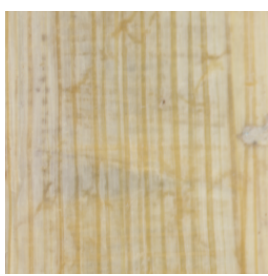

(f) papyrus-P2

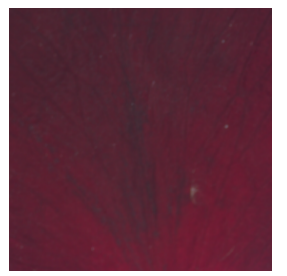

(i) rose petal-R1

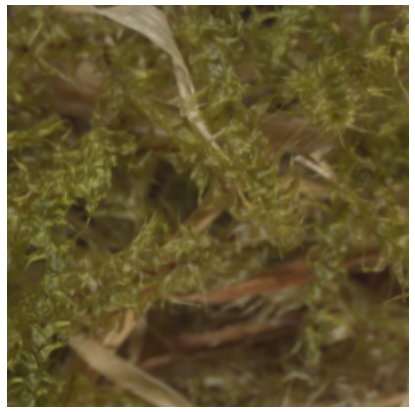

(j) moss-M1

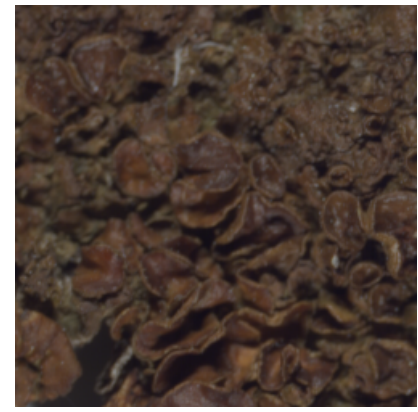

(k) lichen-L6
Fig. 3. Hyperspectral texture dataset used in the experiment. Each image is of $250 \times 250$ pixels and 186 spectral bands in the range of 405.37-995.83 $\mathrm{nm}$. The color images are generated from the hyperspectral images using CIE CMF $2^{\circ}$ observer and D65 illuminant. 


\section{B. Results and analysis}

From the dataset in Fig. 3, we can see that there are some pairs of texture images that, by visual observation, seem to have similar color averages. As an example, the pair of textures L2 (Fig. 3h) and L6 (Fig. 3k) with their dark brown colors. To observe their actual spectral variability, the median spectrum of each texture image is computed. Through these median spectra, we then obtain their respective inner BHSDs in Fig. 4 and inner variance-covariance matrices below:

$$
\widetilde{\Gamma}_{\mathcal{S}_{\mathrm{L} 2}, \widetilde{\mu}_{\mathrm{L} 2}}=\left(\begin{array}{ll}
0.028 & 0.031 \\
0.031 & 0.087
\end{array}\right) \quad \widetilde{\Gamma}_{\mathcal{S}_{\mathrm{L},}, \widetilde{\mu}_{\mathrm{L} 6}}=\left(\begin{array}{ll}
3.44 & 5.32 \\
5.32 & 11.7
\end{array}\right)
$$

As seen from the inner variance-covariance matrices, distribution of spectra within each image differ significantly. Observing the inner BHSD of L2 in Fig. 4, we can see that most of the pixels in this image are contained within the ellipses that visualize bivariate normal law in the representation. Note that the difference between one ellipse and the next is one variancecovariance unit (or standard deviation in the univariate case). On the other hand, spectral variability of L6 is above 3 and it is approximately 100 times larger than that of L2. From the BHSD of L6, roughly two groups of pixels can be observed. The first group is the one that is in the direction of the long axis of the plotted ellipses. Then, another one can be seen as the group of pixels that has a more vertical direction or along $y$-axis of the BHSD. Finally, it can be said that for L6, the modeling through a bivariate normal law that is induced by the spectral MD is not fully suitable.

A BHSD is constructed by the two independent components of KLPD, i.e., shape and intensity differences. Total KLPD, which sums up the two components, allows us to produce a distance map for every single pixel in image. Total KLPD maps for L2 and L6 are provided in Fig. 5, with identical dynamic ranges up to approximately 27 . First of all, since they are presented under identical dynamic ranges, in this representation it is also evident that spectral variability of L6 is larger than L2. This is due to physical variations of the surface being captured. L2 is a capture of one single dry leaf, which can be considered as relatively flat. On the other hand, L6 is a capture of lichen that in itself is composite organisms and composed of objects having different sizes and heights, inducing areas with dark colors or shadow. MD maps in Fig. 5 then allows us to better perceive the variations of texture inside L2, using a standard dynamic range of a normal distribution.

\section{Spectral Statistics For SPeCtral TEXTURE Discrimination}

\section{A. Experimental setup}

The experiment workflow is as shown in Fig. 6. The similarity of a target texture image will be computed through its average MD to the model of a reference texture image. For the reference texture image $\mathcal{S}$, its texture information will be modeled through its median $\widetilde{\mu}_{\mathcal{S}}$, inner BHSD, and inner variance-covariance matrix $\widetilde{\Gamma}_{\mathcal{S}, \widetilde{\mu}_{\mathcal{S}}}$. This part of the experiment is what has been provided and discussed in Section IV.
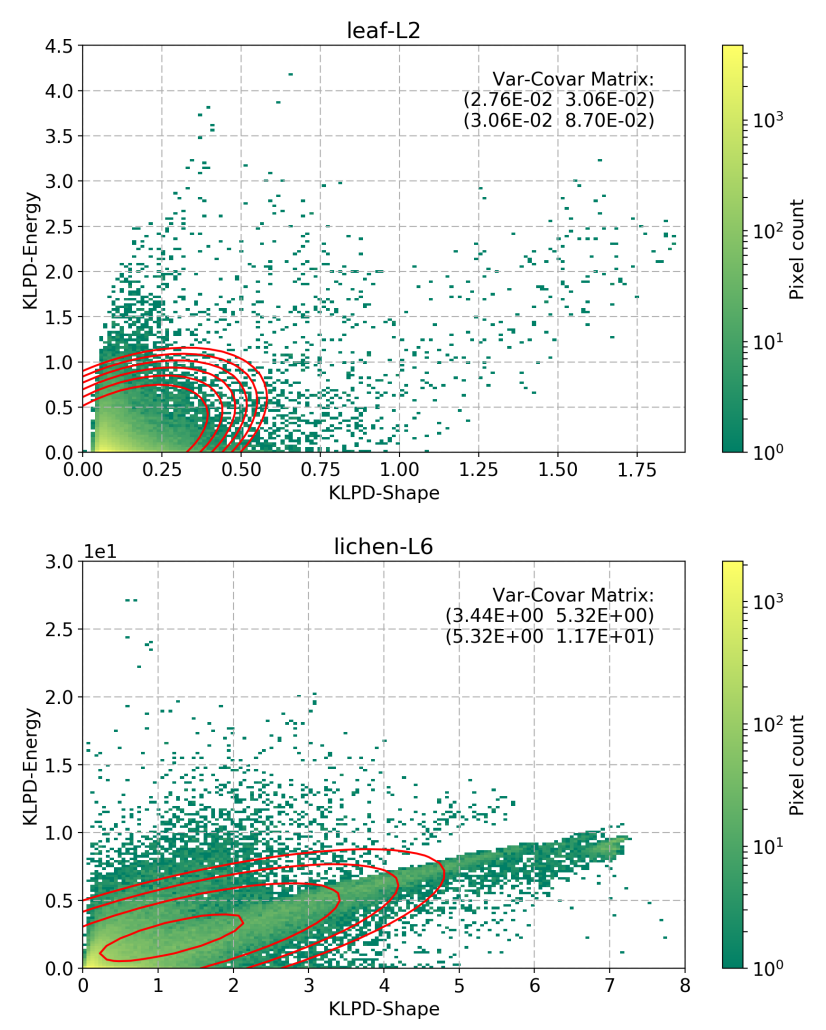

Fig. 4. Inner BHSDs of L2 and L6. Despite visually having similar color averages, their spectral variations are significantly different, see also the dynamic ranges of each variance-covariance matrix.

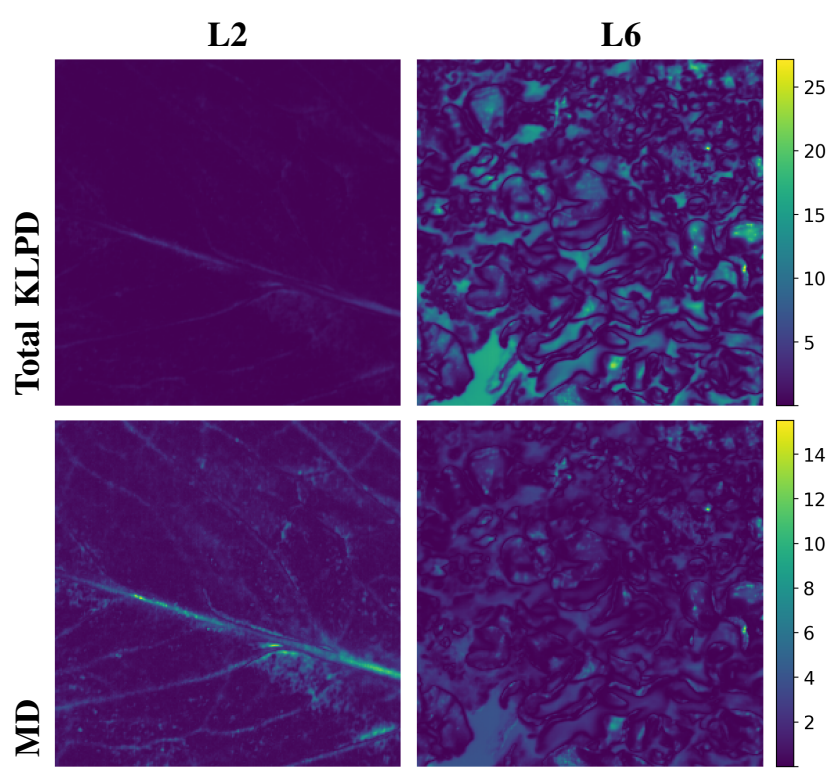

Fig. 5. Spectral difference (KLPD) and spectral Mahalanobis distance (MD) maps of L2 and L6, relative to each inner variance-covariance matrices and spectral medians. KLPD and MD maps each share identical dynamic ranges.

The last step of this texture discrimination application is the decision step, which includes a thresholding. Average MD is not symmetric, since it measures the distance between a spectrum and a distribution. Thus, to determine whether 


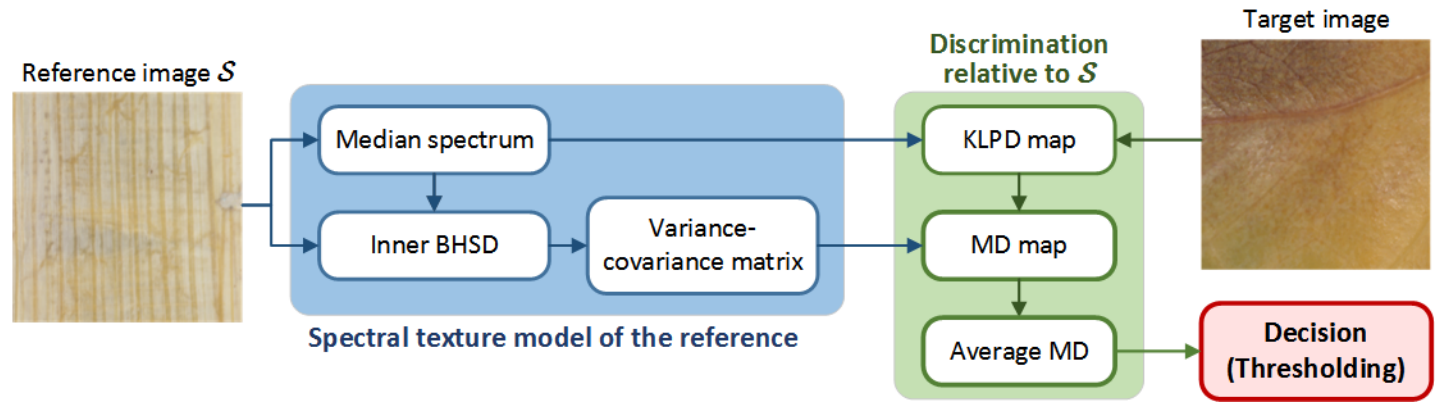

Fig. 6. Experiment workflow for the use of spectral statistics in spectral texture discrimination. For every target image, its average Mahalanobis distance (MD) to every reference texture image $\mathcal{S}$ will be computed, followed by a decision/ thresholding step.

texture image $i$ and $j$ belongs to the same category, we take the maximum of average MD $\bar{d}_{\mathrm{M}}$, see Eq. 7. After obtaining this maximum value, a threshold of 3 standard unit of a bivariate normal distribution is applied to provide a decision.

$$
d\left(\mathcal{S}_{i}, \mathcal{S}_{j}\right)=\max \left(\bar{d}_{\mathrm{M}}\left(S_{i}, \mathcal{S}_{j}\right), \bar{d}_{\mathrm{M}}\left(S_{j}, \mathcal{S}_{i}\right)\right)
$$

\section{B. Results and analysis}

Average MD between pairs of target and reference texture images from the dataset in Fig. 3 is provided in Table I. In this table, images in the row and column are target and reference images, respectively. It can be observed that the similarity of an image to itself is always under 1.5 units, see green colored diagonal cells in the table. There are also instances where one texture is considered similar to the other since its average MD to the reference is between 1.5 and 3 units (light green colors). The red cell colors say that the target image is definitively different from the reference, with average MD above 50 units.

One immediate observation that can be taken is that these average MD values are not symmetric. E.g., average MD of $\mathrm{L} 2$ relative to $\mathrm{M} 1$ is 0.8 . However, for $\mathrm{M} 1$ relative to $\mathrm{L} 2$ it is 15.9 standard units. These results are not unexpected. If we observe the inner BHSDs of L2 (Fig. 4) and M1 (Fig. 7), it is evident that the variability of M1 is larger than L2. Thus, L2 is expected to be more similar to M1 rather than M1 to L2. To also observe the variability within each texture images, their MD maps relative to each other are provided in Fig. 8.

The last step of this texture discrimination application is the decision and thresholding steps, which generate the results in Table II. Through this table, we can see that each texture image is only being attributed to itself, due to having maximum average MD of less than 3 standard units. Furthermore, none of the different images are being considered as similar to the other. Finally, we can conclude that the proposed spectral statistics have allowed achieving good performance in texture discrimination using a simple thresholding step.

\section{CONCLUding REMARKS}

Mahalanobis distance (MD) allows to use normalized threshold for discrimination purposes. According to a model of multivariate normal law, $99.7 \%$ of the spectra from a distribution are at a distance lower than 3. According to the obtained distances and to the asymmetry of similarity

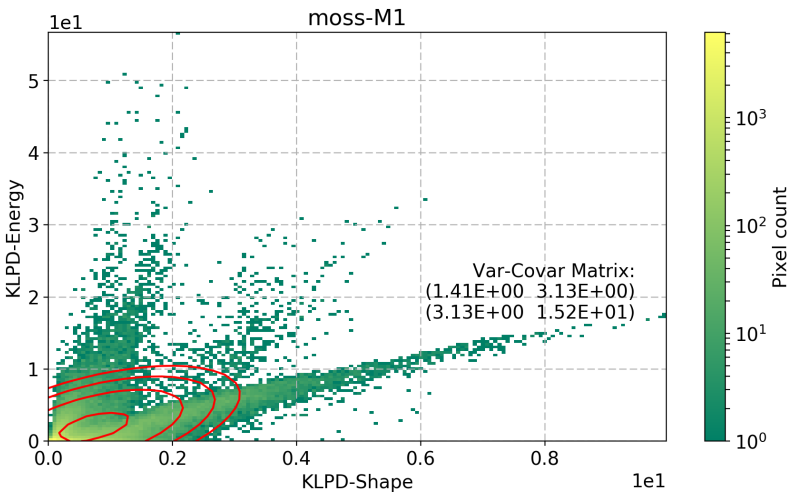

Fig. 7. Inner BHSD of M1, showing that a bivariate normal distribution model does not fit the spectral variability within the image.

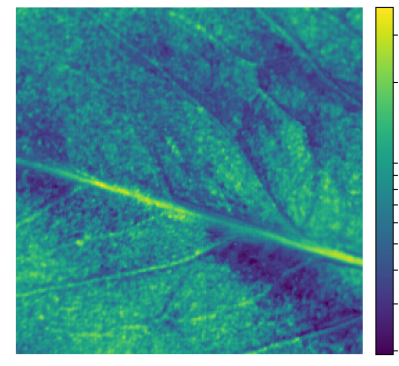

(a) $\mathrm{L} 2$ relative to $\mathrm{M} 1$

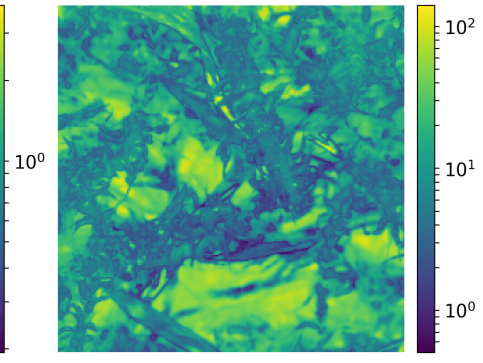

(b) M1 relative to L2
Fig. 8. MD maps of L2 and M1, computed relative to the model of each other, presented in a log colormap.

measures between two textures, the texture discrimination task becomes direct. These preliminary results validate the proposed spectral MD.

Under the texture point of view, the proposed approach is only based on the analysis of spectral variations. It does not take into account the spatial arrangement of spectra within the image. Nevertheless, this study shows the great potential to model a hyperspectral image of a given texture using the spectral median of the image and a variance-covariance matrix processed in the bidimensional histogram of spectral differences (BHSD) space. Our current work continues in the construction of the proposed hyperspectral texture image database, where majority of the images come from the nature. 
TABLE I

AVERAGE MAHAlANOBIS Distance (MD) OF EACH TEXTURE IMAGE $i$ RELATIVE TO THE MODEL OF REFERENCE TEXTURE IMAGE $j$. AVERAGE MD OF AN IMAGE TO ITSELF IS ALWAYS UNDER 1.5 VARIANCE-COVARIANCE UNIT (GREEN COLORS). AN IMAGE IS RELATIVELY SIMILAR TO ANOTHER IF THE VALUE IS BETWEEN 1.5-3 UNITS (LIGHT GREEN), AND DEFINITIVELY DIFFERENT ABOVE 50 UNIT (RED). NOTE THAT THIS TABLE IS ASYMMETRIC.

\begin{tabular}{|c|c|c|c|c|c|c|c|c|c|c|c|c|}
\hline & \multicolumn{11}{|c|}{ Relative to the distribution of image } \\
\hline & & T1 & T2 & T3 & T4 & P1 & $\mathbf{P 2}$ & L1 & L2 & M1 & $\mathbf{R 1}$ & L6 \\
\hline \multirow{11}{*}{ 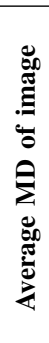 } & T1 & 1.1 & 11.9 & 2.6 & 4.4 & 81.4 & 7.3 & 5.8 & 62.3 & 9.1 & 33.9 & 27.2 \\
\hline & T2 & 11.9 & 1 & 2.9 & 4.3 & 87.2 & 11.9 & 4.7 & 15.3 & 1.7 & 24.5 & 4.5 \\
\hline & T3 & 9 & 3.2 & 1.1 & 2.1 & 110.6 & 12.9 & 4.6 & 21.5 & 3.5 & 21.5 & 12 \\
\hline & T4 & 31.3 & 13.2 & 7.4 & 1.1 & 230.9 & 24.1 & 11 & 41.8 & 5.9 & 7.3 & 10.7 \\
\hline & P1 & 69.2 & 49.7 & 23.1 & 29.1 & 1.2 & 4.1 & 18.8 & 178.5 & 20.3 & 93.2 & 55.6 \\
\hline & P2 & 55.7 & 46.3 & 20.1 & 25 & 11.8 & 1.1 & 13 & 90.4 & 12.1 & 109.8 & 29.2 \\
\hline & L1 & 10.2 & 12.3 & 4.3 & 6.3 & 68.3 & 6.4 & 1.1 & 17.5 & 3.2 & 46.2 & 11.6 \\
\hline & L2 & 24.9 & 9.4 & 6.1 & 5.8 & 102.8 & 13 & 4.2 & 1 & 0.8 & 23.6 & 4.8 \\
\hline & M1 & 21.8 & 8.2 & 7.8 & 8.8 & 89.6 & 13.2 & 6.6 & 15.9 & 0.9 & 53 & 2.4 \\
\hline & R1 & 61.1 & 26.9 & 15.8 & 3 & 324.3 & 34.2 & 20.7 & 62.4 & 9.1 & 1.1 & 9.5 \\
\hline & L6 & 28.8 & 15.8 & 15 & 16.7 & 114.8 & 19.5 & 14.9 & 47.9 & 3.2 & 117.7 & 1 \\
\hline
\end{tabular}

TABLE II

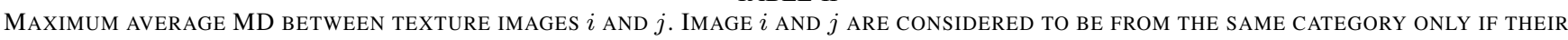
MAXIMUM AVERAGE MD IS BELOW THE THRESHOLD OF 3 STANDARD UNIT OF BIVARIATE NORMAL DISTRIBUTION LAW (GREEN COLORED CELLS).

\begin{tabular}{|c|c|c|c|c|c|c|c|c|c|c|c|}
\hline & \multicolumn{11}{|c|}{ Maximum average MD between pairs of images } \\
\hline & T1 & T2 & T3 & T4 & P1 & $\mathbf{P 2}$ & L1 & $\mathbf{L 2}$ & M1 & R1 & L6 \\
\hline T1 & 1.1 & 11.9 & 9 & 31.3 & 81.4 & 55.7 & 10.2 & 62.3 & 21.8 & 61.1 & 28.8 \\
\hline T2 & & 1 & 3.2 & 13.2 & 87.2 & 46.3 & 12.3 & 15.3 & 8.2 & 26.9 & 15.8 \\
\hline T3 & & & 1.1 & 7.4 & 110.6 & 20.1 & 4.6 & 21.5 & 7.8 & 21.5 & 15 \\
\hline T4 & & & & 1.1 & 230.9 & 25 & 11 & 41.8 & 8.8 & 7.3 & 16.7 \\
\hline P1 & & & & & 1.2 & 11.8 & 68.3 & 178.5 & 89.6 & 324.3 & 114.8 \\
\hline P2 & & & & & & 1.1 & 13 & 90.4 & 13.2 & 109.8 & 29.2 \\
\hline L1 & & & & & & & 1.1 & 17.5 & 6.6 & 46.2 & 14.9 \\
\hline L2 & & & & & & & & 1 & 15.9 & 62.4 & 47.9 \\
\hline M1 & & & & & & & & & 0.9 & 53 & 3.2 \\
\hline R1 & & & & & & & & & & 1.1 & 117.7 \\
\hline L6 & & & & & & & & & & & 1 \\
\hline
\end{tabular}

\section{REFERENCES}

[1] A. F. Goetz, G. Vane, J. E. Solomon, and B. N. Rock, "Imaging spectrometry for earth remote sensing," Science, vol. 228, no. 4704, pp. 1147-1153, 1985.

[2] G. Elmasry, M. Kamruzzaman, D.-W. Sun, and P. Allen, "Principles and applications of hyperspectral imaging in quality evaluation of agrofood products: A review," Crit Rev Food Sci Nutr, vol. 52, no. 11, pp. 999-1023, 2012.

[3] R. Siche, R. Vejarano, V. Aredo, L. Velasquez, E. Saldaña, and R. Quevedo, "Evaluation of food quality and safety with hyperspectral imaging (HSI)," Food Eng Rev, vol. 8, no. 3, pp. 306-322, 2016.

[4] C. Wang, W. Zheng, Y. Bu, S. Chang, S. Zhang, and R. X. Xu, "Multiscale hyperspectral imaging of cervical neoplasia," Arch Gynecol Obstet, vol. 293, no. 6, pp. 1309-1317, 2016.

[5] A. Haddadi, B. Leblon, Z. Pirouz, J. Nader, and K. Groves, "Prediction of wood properties for thawed and frozen logs of quaking aspen, balsam poplar, and black spruce from near-infrared hyperspectral images," Wood Sci Technol, vol. 50, no. 2, pp. 221-243, 2016.

[6] T. Adão, J. Hruška, L. Pádua, J. Bessa, E. Peres, R. Morais, and J. J. Sousa, "Hyperspectral imaging: A review on UAV-based sensors, data processing and applications for agriculture and forestry," Remote Sens, vol. 9, no. 11, 2017.

[7] JCGM-Joint Committee for Guides in Metrology, "International vocabulary of metrology-Basic and general concepts and associated terms," http://goo.gl/AJIIoz, Tech. Rep., 2012.

[8] H. Deborah, N. Richard, and J. Y. Hardeberg, "A comprehensive evaluation on spectral distance functions and metrics for hyperspectral image processing," IEEE J Sel Topics Appl Earth Observ Remote Sens, vol. 8, no. 6, pp. 3224-3234, 2015.

[9] N. Richard, D. Helbert, C. Olivier, and M. Tamisier, "Pseudo-divergence and bidimensional histogram of spectral differences for hyperspectral image processing," J Imaging Sci Technol, vol. 60, no. 5, pp. $50402-$ $1-50402-13,2016$

[10] H. Deborah, N. Richard, J. Y. Hardeberg, and C. Fernandez-Maloigne, "Assessment protocols and comparison of ordering relations for spectral image processing," IEEE J Sel Topics Appl Earth Observ Remote Sens, vol. 11 , no. 4, pp. 1253-1265, 2018.

[11] H. Deborah, "Towards spectral mathematical morphology," phdthesis, Norwegian University of Science \& Technology, University of Poitiers, Dec 2016. [Online]. Available: http://goo.gl/AsfCjj

[12] N. Richard, H. Deborah, and O. Ben-Ahmed, "Hyperspectral image processing: Full-band, dimensionality reduction, or band selection approach?" in Treatment and Analysis of the Information Methods and Applications (TAIMA), 2018.

[13] S. Kullback and R. A. Leibler, "On information and sufficiency," Annals of Mathematical Statistics, vol. 22, no. 1, pp. 79-86, 1951.

[14] M. D. Mura, J. A. Benediktsson, B. Waske, and L. Bruzzone, "Morphological attribute profiles for the analysis of very high resolution images," IEEE Trans Geosci Remote Sens, vol. 48, no. 10, pp. 3747-3762, 2010.

[15] A. Zare and K. C. Ho, "Endmember variability in hyperspectral analysis: Addressing spectral variability during spectral unmixing," IEEE Signal Process Mag, vol. 31, no. 1, pp. 95-104, 2014.

[16] L. Drumetz, J. Chanussot, and C. Jutten, "Variability of the endmembers in spectral unmixing: Recent advances," in Hyperspectral Image and Signal Processing: Evolution in Remote Sensing (WHISPERS), 8th Workshop on, 2016, pp. 1-5.

[17] D. Manolakis, D. Marden, J. Kerekes, and G. Shaw, "On the statistics of hyperspectral imaging data," in Proc. SPIE: Algorithms for Multispectral, Hyperspectral, and Ultraspectral Imagery VII, vol. 4381, 2001, pp. 308-316.

[18] A. Mirhashemi, "Introducing spectral moment features in analyzing the spectex hyperspectral texture database," Mach Vis Appl, 2017. 